\title{
Genome scale CRISPR Cas9a knockout screen reveals genes that controls glioblastoma susceptibility to the alkylating agent temozolomide.
}

Chidiebere U Awah ${ }^{1,2+}$, Jan Winter ${ }^{4}$, Olorunseun O. Ogunwobi ${ }^{1,2,3,}$

1 Department of Biological Sciences, Hunter College of The City University of New York, New York, NY, USA

2 Joan and Sanford I. Weill Department of Medicine, Weill Cornell Medicine, Cornell University, New York, NY, USA

3 Hunter College for Cancer Health Disparities Research, Hunter College of The City University of New York, New York,

4 Steinbrenner Laborsysteme GmbH, Wiesenbach, Germany.

+ Correspondence should be addressed to

Chidiebere U Awah MD MSc PhD

Department of Biological Sciences, Hunter College of The City University of New York, New York, NY, USA

Email: cha4010@med.cornell.edu 


\begin{abstract}
Glioblastoma is the most fatal of all primary human brain tumors with 14 months survival, at best. The mainstay therapy for this tumor involves temozolomide, surgery, radiotherapy and tumor treating electric field. Cancer resistance to commonly available chemotherapeutics remains a major challenge in glioblastoma patients receiving treatment and unfavorably impact their overall survival and outcome. However, the lack of progress in this area could be attributed to lack of tools to probe unbiasedly at the genome wide level the coding and non-coding elements contribution on a large scale for factors that control resistance to chemotherapeutics. Understanding the mechanisms of resistance to chemotherapeutics will enable precision medicine in the treatment of cancer patients.

CRISPR Cas9a has emerged as a functional genomics tool to study at genome level the factors that control cancer resistance to drugs. Recently, we used genome wide CRISPR-Cas9a screen to identify genes responsible for glioblastoma susceptibility to etoposide. We extended our inquiry to understand genes that control glioblastoma response to temozolomide by using genome scale CRISPR. This study shows that the unbiased genome-wide loss of function approach can be applied to discover genes that influence tumor resistance to chemotherapeutics and contribute to chemoresistance in glioblastoma.
\end{abstract}


bioRxiv preprint doi: https://doi.org/10.1101/2021.08.11.455972; this version posted August 12, 2021. The copyright holder for this preprint (which was not certified by peer review) is the author/funder. All rights reserved. No reuse allowed without permission.

\section{Introduction}

Glioblastoma is the deadliest of all primary brain tumors with a very poor survival outcome. Temozolomide, an alkylating agent, is used as a mainstay drug in treating of glioblastoma patients along with radiation, tumor treating field and surgery ${ }^{1,2}$. However, this drug offers little to no benefit for glioblastoma patients. Hence, the objective of the generation of this data is to understand and uncover genes that control glioblastoma susceptibility and resistance to temozolomide using an unbiased genome scale CRISPR Cas9a knockout screen. 


\section{Material and Methods \\ Details of Source of all samples}

Cell lines: The glioma cell line, U251 was commercially obtained from ATCC and used in the genome scale CRISPR screen.

Reagent: The U251 cells were grown in 10\% FBS DMEM media with antibiotics up to $80 \%$ confluency before downstream analysis.

CRISPR Knockout library: The Brunello library pooled gRNA (Cat no: 73178-LV) were commercially purchased from Addgene. This library contains 70,000sgRNA, with 60,000 guides targeting approximately the 20,000 genes in the human genome at the rate of 3-4 guides per gene. There are about 10,000 guides which are the non-targeting controls.

Determination of viral titre and multiplicity of infection: $3 \times 10^{6}$ of U251 cells are seeded into 12 -well plate in $2 \mathrm{ml}$. The Brunello library as virus supernatant were added at $400 \mu \mathrm{l}, 200 \mu \mathrm{l}, 100 \mu \mathrm{l}$, $75 \mu \mathrm{l}, 50 \mu \mathrm{l}, 25 \mu \mathrm{l}$ and $8 \mu \mathrm{g} / \mu \mathrm{l}$ of polybrene is and spinfected at $1000 \mathrm{~g}$ at $33^{\circ} \mathrm{C}$ for $2 \mathrm{hrs}$. Cells then are incubated at $37^{\circ} \mathrm{C}$. After $24 \mathrm{hrs}$ the cells are harvested and seeded at $4 \times 10^{3}$ with puromycin for 96hrs with a well containing cells that were not transduced with any virus. After $96 \mathrm{hrs}$ the cell titre glo (Promega cat is used to determine cell viability at MOI $21 \%$. At the multiplicity of infection (MOI) of $21 \%$ we are able to infect 1 sgRNA/cell (Figure 1A-C).

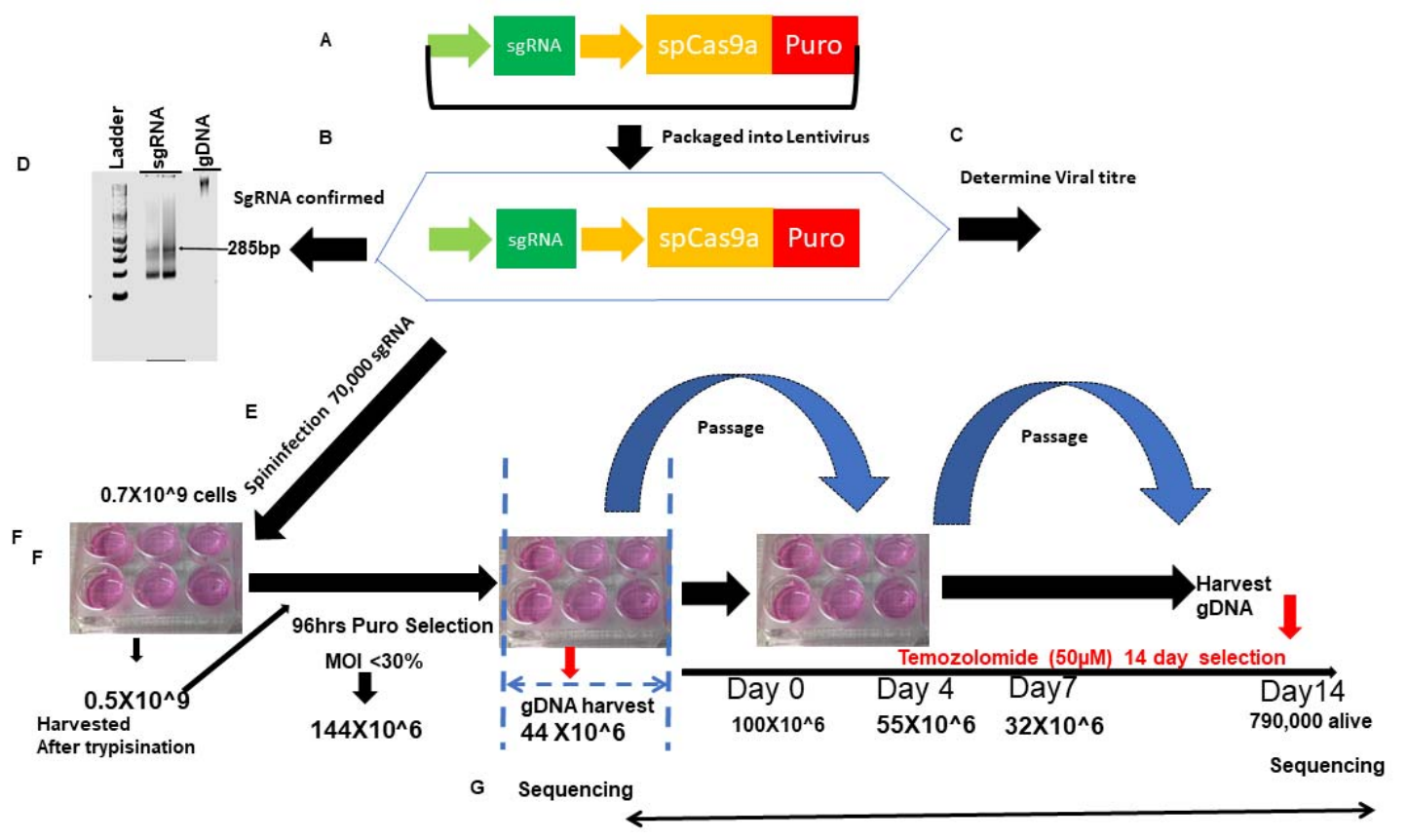

Figure 1: Strategy for genome scale CRISPR screen. A. shows the schematic depiction of the sgRNA library B. Library is packaged into lentiviral. $\mathrm{C}$ viral titre and $\mathrm{MOI}$ is determined. D A gel shows amplification of sgRNA library. E Lentivirus containing sgRNA is spinfected into glioma cells. F Transduced cells are selected with puromycin after which some cells are harvested for baseline sgRNA representation, the rest of the cells are placed under the treatment of temozolomide/DMSO/puromycin for 14 days. G. cells are harvested, sgRNA extracted and sequenced on NEXT generation sequencers. 
Genome wide scale CRISPR-Cas9a knockout screen: To do the CRISPR screen, we expanded the U251 to 500million cells and then spinfected with 70,000sgRNA. The library was integrated into the cells by spinfection, and subsequently selected with $0.6 \mu \mathrm{g} / \mathrm{ml}$ of puromycin for 4days. This selection is aimed at the cells that have been rightly integrated with the sgRNA that incorporates the puromycin cassette into their genome. We achieved an $\mathrm{MOI}$ of $21 \%$ in two independent screens. At the end of day 4 , about 150 million cells survived the selection. We used 50 million of selected cells for the extraction of genomic DNA. The base sgRNA representation is obtained by amplification of the sgRNA with unique barcoded primers. The remaining 100 million cells were expanded for 2days once cells grew to 300 million. 100 million of cells were treated with temozolomide at concentration of $50 \mu \mathrm{M}$ for 14 days, and the 100 million each were treated with DMSO and Puromycin for 14 days respectively and served as control. DMSO is control for temozolomide treated cells, whereas puromycin is for identification of essential genes driving glioma After 14days, the cells were harvested, the gDNA extracted, and the sgRNA amplified with another unique barcoded primer (Figure 1D- G; Figure 2 A, Figure 3 A-B).

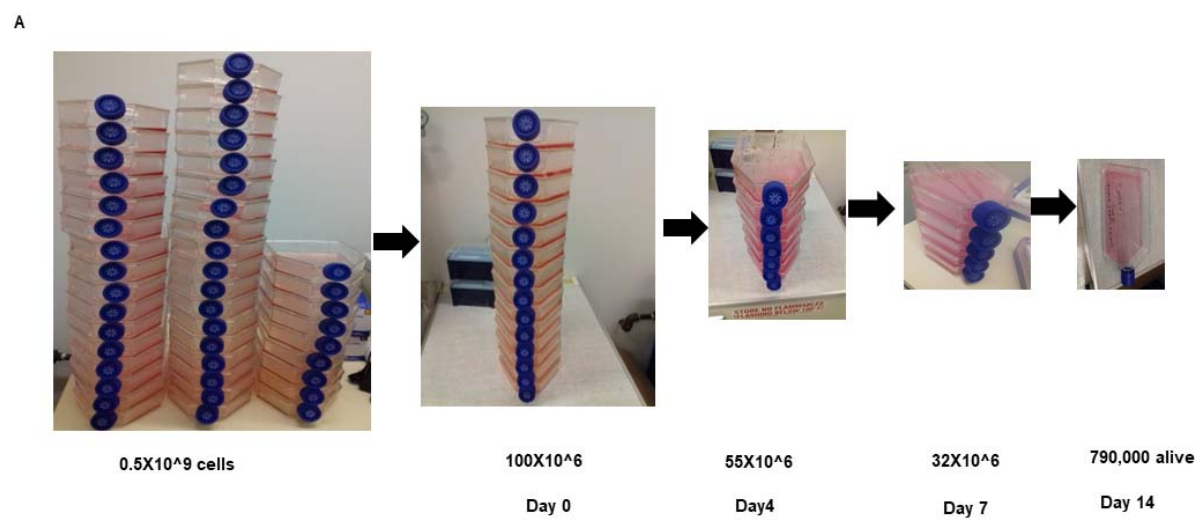

Figure 2: Kinetics of cell selection pressure under chemotherapy in genome scale CRISPR screen. A. shows the schematic depiction of number of T225 flask of cell starting from over half a billion cells to the number that remains after 14 days of selection.
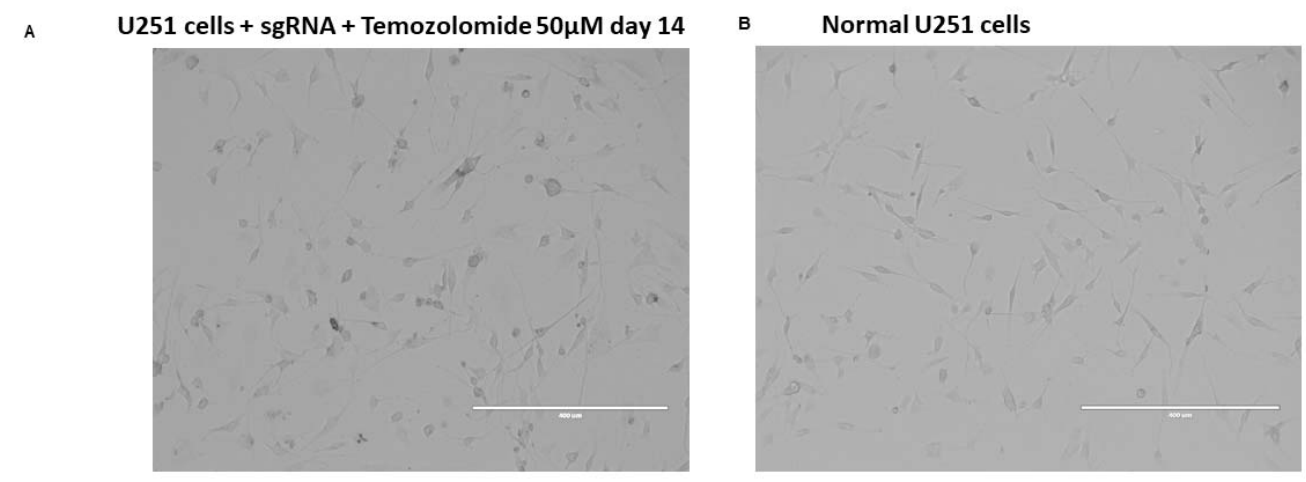

Figure 3: Morphology of cells transduced with CRISPR library under drug selection compared to wildtype control. A. shows U251 cells transduced with sgRNA treated with temozolomide for 14 days and B shows normal U251 wildtype controls. 
DNA extraction and PCR amplification of pooled sgRNA: The genomic DNA (gDNA) were extracted with the Zymo Research Quick-DNA midiprep plus kit (Cat No: D4075). gDNA was further cleaned by precipitation with $100 \%$ ethanol with $1 / 10$ volume $3 \mathrm{M}$ sodium acetate, $\mathrm{PH} 5.2$ and 1:40 glycogen co-precipitant (Invitrogen Cat No: AM9515). The gDNA concentration were measured by Nano drop 2000 (Thermo Scientific). The PCR were set up as:

\section{$50 \mu$ reaction}

$5 \mu$ of $10 x$ buffer

$4 \mu \mathrm{l}$ of $50 \mathrm{mM}$ DNTP $(1 / 25)$

$0.5 \mu \mathrm{l}$ of P5 forward primers equimolar mix

$10 \mu \mathrm{l}$ of $20 \mu \mathrm{M}$ reverse primer (1 per experimental condition)

gDNA (20ng for sgRNA library) or $2-10 \mu g$ for sgRNA in various the experimental condition

$1.5 \mu$ l of Extaq enzyme

$27 \mu \mathrm{l}$ of $\mathrm{H} 20$

\section{With NEBNEXT PCR polymerase enzyme the following recipe is used:}

$25 \mu$ l of NEB Next High Fidelity Master Mix (2x)

$1 \mu \mathrm{l}$ of gDNA (20ng for sgRNA library) or $2-10 \mu \mathrm{g}$ for sgRNA in various the experimental condition

$1.25 \mu$ l of P5 forward primers equimolar mix

$1.25 \mu \mathrm{l}$ of $20 \mu \mathrm{M}$ reverse primer (1 per experimental condition)

21.5 $\mu$ l ultrapure $\mathrm{H} 20$

The sequences of P5 forward primers and the reverse primers can be found described ${ }^{14}$.

The PCR cycle used is:

$\begin{array}{clcc}\text { Cycle number } & \text { Denature } & \text { Anneal } & \text { Extend } \\ 1 & 98^{\circ} \mathrm{C} 3 \mathrm{mins} & & \\ 2-23 & 98^{\circ} \mathrm{C} 10 \mathrm{sec} & 63^{\circ} \mathrm{C} 10 \mathrm{sec} & 72^{\circ} \mathrm{C} 2 \mathrm{mins}\end{array}$

Don't exceed 23 cycles. We have found that difficulty gDNA containing sgRNA can be amplified using annealing temperature gradient of $53-68^{\circ} \mathrm{C}$. The sgRNA knockout size is $285 \mathrm{bp}$. All extracted gDNA from the cells were all amplified to maintain the library coverage. 
A

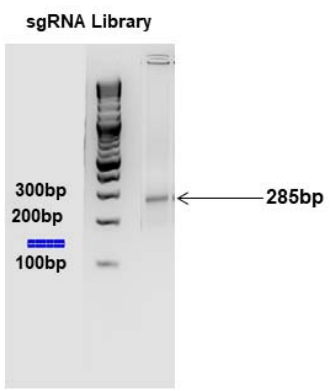

D

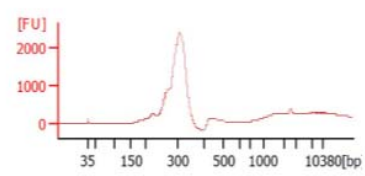

B sgRNA from Puromycin and DMSO selection

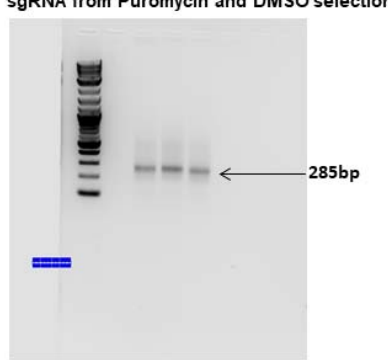

E

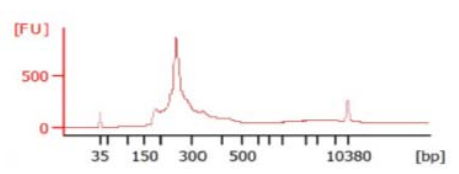

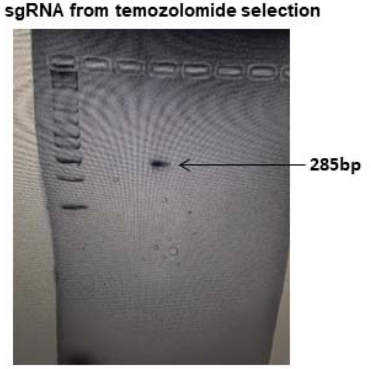

F

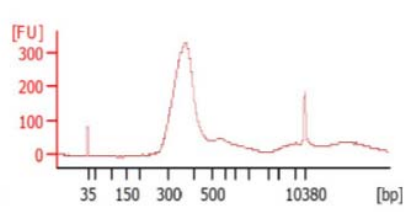

Figure 4: PCR amplification of sgRNA from library, puromycin, DMSO and temozolomide selected glioblastoma: A-C shows agarose gel pic of the sgRNA amplified from library, puromycin, DMSO and temozolomide treated cells. D-F shows electropherogram measurement of the amplified sgRNA from the different conditions. 
Next Generation Sequencing. The sgRNAs amplified and gel extracted from Figure 4 were pooled together and sequenced in a Next generation sequencer (Next Seq) at 300 million reads for the four sgRNA pool aiming at 1,000reads/sgRNA. The samples were sequenced according to the Illumina user manual with 80 cycles of read 1 (forward) and 8 cycles of index $1^{3,4} .20 \%$ PhiX were added on the Next Seq to improve library diversity and aiming for a coverage of $>1000$ reads per SgRNA in the library.

CRISPR screen data analysis. All data analysis was performed with the bioinformatics tool CRISPR Analyzer (http://crispr-analyzer.dkfz.de/) $)^{5}$.

The sequence reads obtained from Next Seq as fastq files were aligned with human genome in quality assessment to determine the percentage that map to the total human genome (Figure 5).

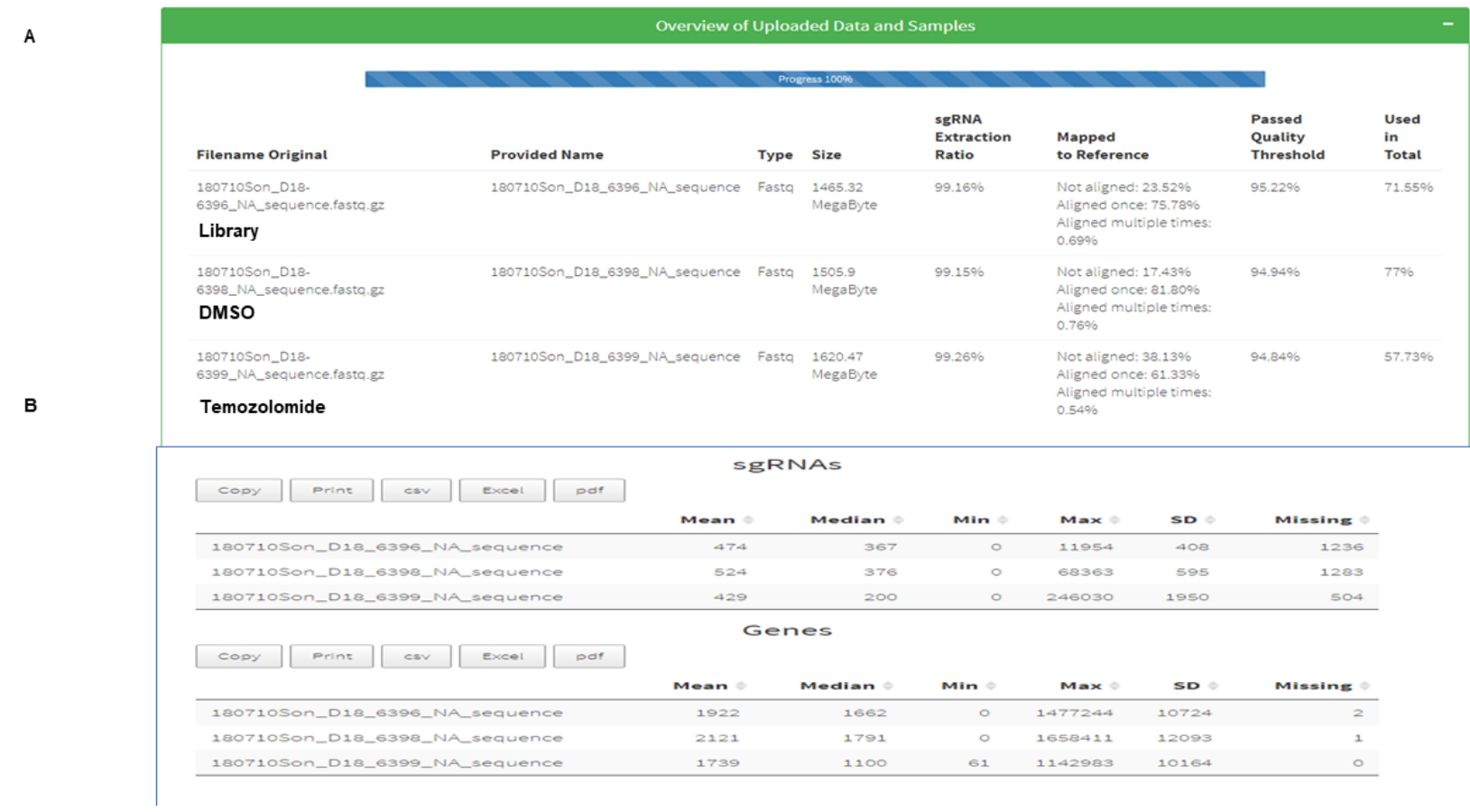

Figure 5: Alignment of Next Seq sgRNA reads from library, DMSO and Temozolomide treated cells. A show the mapping of sgRNA library (75.78\%) DMSO (81.8\%) and temozolomide (61.33\%) mapped to the human reference genome. B. shows the number genes and sgRNA missing from the mapped library which were few.

To find genes that were enriched or depleted under various treatment condition, the CRISPRAnalyzer performs four rigorous statistical analysis where are (1) DeSeq 2 (Log2 fold sgRNA count) 3 (Wilcoxon t-test) and 4 (Z-ratio) comparing the enrichment of guides in treatment conditions versus their non-targeting controls. $P$ value of 0.05 is used as a cut off for guides statistical enrichment. The sgRNA that does not meet a read count of 20 is removed. Hit calling from the CRISPR screen was done based on sgRSEA enriched, $p<0.01$ was used for significance based on Wilcoxon test. 
To set up the analysis, the sgRNA reads (library, puromycin, DMSO and temozolomide) replicates were loaded unto the software. The sgRNA that does not meet a read count of 20 is removed. Hit calling from the CRISPR screen was done based on sgRSEA enriched, $p<0.01$ was used for significance based on Wilcoxon test.

CRISPR Analyzer does also correlation analysis based on Pearson and Spearman statistical analysis to determine the relatedness between samples replicates and then uses log2 fold SgRNA read count to calculate the cumulative frequency between guides in various treated samples compared to controls in cumulative frequency curve (Figure 6) and in scatter plot (Figure 7).

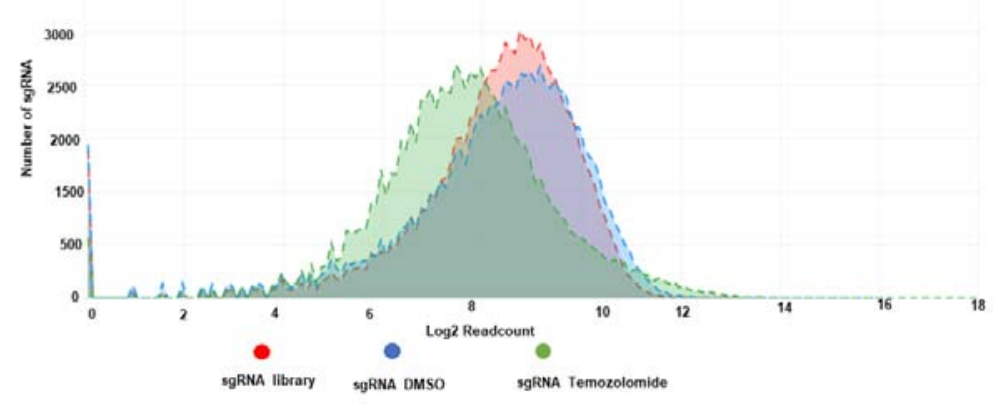

Figure 6: Cummulative frequency of sgRNA distribution in CRISPR library, DMSO and Temozolomide treated glioblastoma cells. A shows cumulative frequency distribution of log 2 sgRNA read count in sgRNA library (red), sgRNA DMSO (blue) and sgRNA Temozolomide (green).

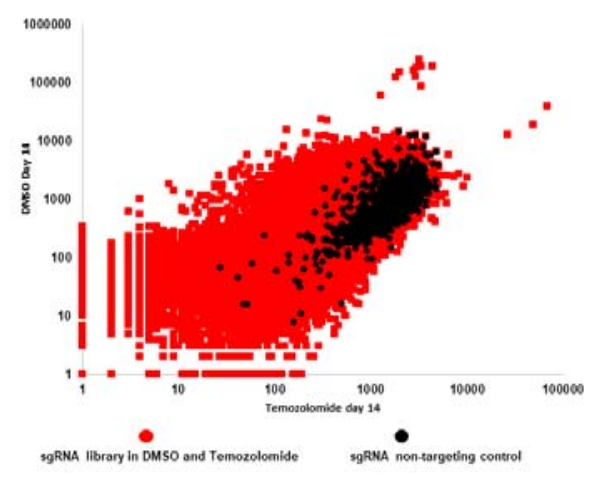


Figure 7: Scatter plot distribution of sgRNA in DMSO versus temozolomide treated glioblastoma cells. A Scatter plot depicting sgRNA distribution in DMSO (y-axis) and Temozolomide (x-axis) and their non-targeting controls (black dots).

\section{Dataset validation}

To validate the data from the screen, we found that NSUN6 a 5mC RNA methyltransferase is enriched in the CRISPR screen under temozolomide compared to DMSO and puromycin selection for 14 days. We performed a single gene editing of NSUN6 and proved that loss of NSUN6 led to loss of $5 \mathrm{mC}$ and caused resistance to temozolomide. This data has been presented in the primary article ${ }^{6}$.

\section{Data availability}

All data underlying the results are available as part of the article and no additional source data are required ${ }^{7}$.

DOI: $10.5281 /$ zenodo.5167143

Data 1: sgRNA library

Data 3: DMSO treated sgRNA library replicate 1

Data 4: DMSO treated sgRNA library replicate 2

Data 5: Puromycin treated sgRNA Library replicate 1

Data 6: Puromycin treated sgRNA library replicate 2

Data 7: Temozolomide treated sgRNA library

\section{Software availability}

CRISPRAnalyzer the software used in this data analysis is publicly available at (http://crispranalyzer.dkfz.de/).

\section{Grant information}

Olorunseun O. Ogunwobi is supported by National Cancer Institute grant \#U54 CA221704.

\section{Competing interest}

Authors declare no competing interest

\section{Acknowledgment}

We acknowledge our colleagues who provided support in the cell culture. 


\section{References}

1. Stupp, R, et al. Effect of Tumor-Treating Fields Plus Maintenance Temozolomide vs Maintenance Temozolomide Alone on Survival in Patients with Glioblastoma: A Randomized Clinical Trial. JAMA, 2017. 318(23): p. 2306-2316.

2. Verhaak, R.G. et al., Integrated genomic analysis identifies clinically relevant subtypes of glioblastoma characterized by abnormalities in PDGFRA, IDH1, EGFR, and NF1. Cancer Cell, 2010. 17(1): p. 98-110.

3. Joung, J., et al., Genome-scale CRISPR-Cas9 knockout and transcriptional activation screening. Nat Protoc, 2017. 12(4): p. 828-863.

4. Awah CU et al (2020) Ribosomal protein S11 influences glioma response to TOP2 poisons. Oncogene 39 (27) 5068-5081

5. Winter. J Schwering M, Pelz O, Rauscher B, Zhan T, Heigwer F, Boutros M (2017). CRISPRAnalyzeR: Interactive analysis, annotation, and documentation of pooled CRISPR screens. BioRxiv 2017.

6. Awah CU et al (2021) NSUN6, an RNA methyltransferase of 5-mC controls glioblastoma response to Temozolomide (TMZ) via NELFB and RPS6KB2 interaction (Under review in Cancer Biology \& Therapy)

7. Awah CU et al (2021) Data set: Genome scale CRISPR Cas9a knockout screen reveals genes that controls glioblastoma susceptibility to the alkylating agent temozolomide (TMZ). DOI: 10.5281/zenodo.5167143 